Información Tecnológica

Vol. 25(1), 15-22 (2014)

doi: $10.4067 / S 0718-07642014000100003$

\title{
Estudio de las Fuerzas de Avance en un Proceso de Taladrado utilizando Brocas con Geometría Mejorada
}

\author{
Mario A. Guzmán y Fernando T. Caire \\ Universidad de La Frontera, Facultad de Ingeniería, Ciencias y Administración, Departamento de Ingeniería \\ Mecánica, Avda. Francisco Salazar 01145, Temuco-Chile. (e-mail: mario.guzman@ufrontera.cl, \\ fernando.caire@ufrontera.cl)
}

Recibido May. 6, 2013; Aceptado Jun. 19, 2013; Versión final recibida Ago. 20, 2013

\begin{abstract}
Resumen
Dos tipos de brocas son sometidas a un proceso de taladrado con la finalidad de determinar cuál diseño de herramienta ofrece una disminución en las fuerzas de avance cuando se taladran agujeros de un mismo diámetro, bajo las mismas condiciones de mecanizado. Una de las brocas estudiadas fue de geometría convencional y otra de geometría mejorada. La captura de las fuerzas de mecanizado se hace mediante una plataforma dinamométrica con sensores piezoeléctricos. Los datos son sometidos a un proceso de filtrado, para posteriormente determinar y graficar el comportamiento experimental y simulado de los procesos de taladrado. Los resultados obtenidos demuestran una disminución de las fuerzas de avance cuando se taladra con brocas mejoradas en la parte activa.
\end{abstract}

Palabras clave: brocas mejoradas, filo transversal, bisel transversal, indentación, fuerzas en taladrado

\section{Study of Thrust Forces in a Drilling Process utilizing drills with Improved Geometry}

\begin{abstract}
Two types of drills were submitted to a drilling process with the objective of determining which tool design offers a decrease in the thrust forces when holes of the same diameter are drilled, under the same mechanizing conditions. One of the drills was with conventional geometry and the other one was with improved geometry. The capture of the mechanized forces is done through a dynamometer platform with piezoelectric sensors. The data is put under a filtering process, to then determine and plot the experimental data and simulated behavior of the drilling processes. The results obtained show a decrease of the thrust forces when drilling with drills that have their active part improved.
\end{abstract}

Keywords: improved drills, transverse edge, chisel edge, indentation, drilling forces 


\section{INTRODUCCIÓN}

Una broca clásica está caracterizada, principalmente, por ángulo de la punta, el ángulo de la hélice, la longitud de los filos principales y la presencia del bisel transversal. Todos ellos, tienen una gran influencia sobre las fuerzas de corte y la calidad de los agujeros (Pirtini y Lazoglu, 2005). Los investigadores (Shaw y Oxford, 1957) establecieron que el proceso de taladrado puede ser dividido en dos partes. Una es la acción de los filos principales de corte y la otra la acción del bisel transversal. En una operación de taladrado el bisel transversal arranca material mediante un proceso de indentación cuyo ángulo de desprendimiento es altamente negativo. La fuerza sobre el bisel que se hace presente en la dirección axial de la broca tiene un valor determinado experimentalmente por (Oxford y Rochester, 1955), cuyo resultado estableció que esta fuerza puede llegar a tener el mismo valor de las fuerzas que se producen sobre los filos principales

Para minimizar la fuerza de avance y el par de mecanizado en un proceso de taladrado, algunos investigadores han logrado en cierta medida, optimizar la geometría de la punta de la herramienta, haciendo algunas leves modificaciones al bisel transversal tradicional (Wang y Zhang, 2008), (Anish et al 2005). Recientemente se ha mejorado la geometría de la punta de las brocas, lo que permite en particular asegurar el autocentrado de la broca, sin tener que utilizar previamente una broca de centrado. Desde el punto de vista de la operación de taladrado, se garantiza un mecanizado más eficaz sobre toda la longitud de la arista de corte, contribuyendo además, a un buen desprendimiento de la viruta y gran duración de la herramienta, incluso en el caso de mecanizado con materiales muy duros.

Las mejoras realizadas en la geometría de las brocas tradicionales (Fig.1), que divide o parte al bisel transversal en dos filos de corte (Fig.2), lo que corresponde a un destalonado en cruz. Esta herramienta mejorada conduce a nuevos procedimientos de estimación de las fuerzas y del par mecánico de taladrado. En ese sentido, este trabajo investigativo ha dado lugar a realizar un estudio específico del proceso de taladrado considerando estas nuevas geometrías de herramientas, donde los parámetros de corte ligados a este tipo de brocas y los análisis matemáticos asociados, están referidos exclusivamente, a lo que sucede en la punta de la herramienta. El autor Audy, (2008) ha presentado un trabajo para predecir las fuerzas de taladrado, considerando algunas modificaciones en la geometría de la punta de las brocas. Específicamente, se puso énfasis en disminuir el largo del bisel transversal tradicional, como también, optimizar el ángulo de desprendimiento de los filos principales. Sin embargo, dichas herramientas han mantenido la presencia del bisel transversal tradicional.
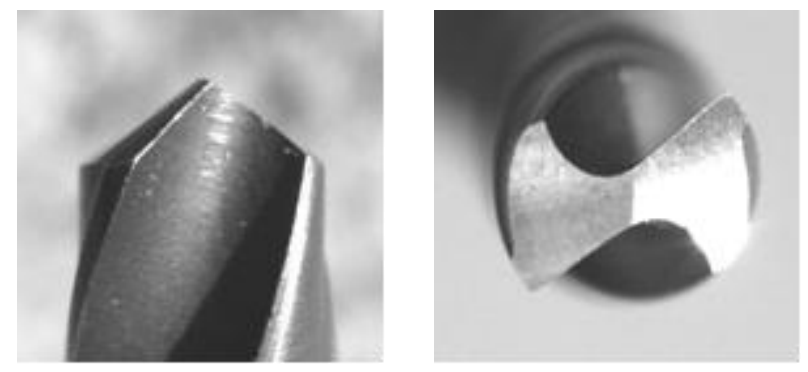

Fig.1: Broca tradicional con bisel transversal
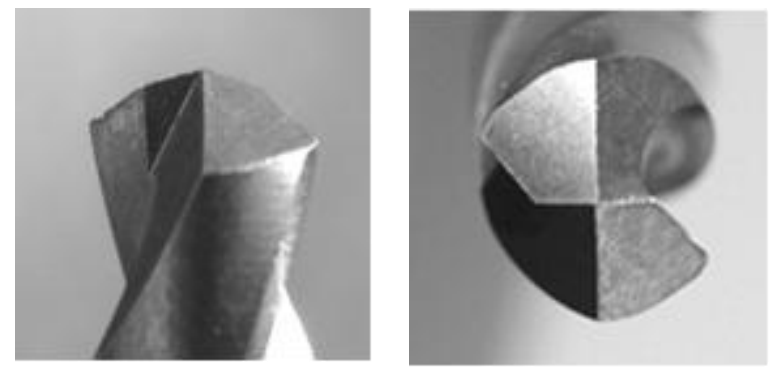

Fig.2: Broca mejorada con filo trasversal.

Las fuerzas principales que participan en un proceso de taladrado son; la fuerza de avance axial y la fuerza de corte. Esta última es como consecuencia del movimiento rotatorio de la herramienta, la que conduce a la generación del par de taladrado. Respecto de la fuerza de avance se puede decir que está constituida por la sumatoria de las fuerzas axiales que actúan en los filos principales y la fuerza que se produce en el filo transversal de la herramienta. Se reconoce que el filo transversal de la broca es una componente significativa de la fuerza de avance. (Hamade et al, 2006).

Un componente importante de la geometría de la herramienta es el bisel transversal, el cual tiene una alta incidencia en la fuerza de avance. El investigador Gong et al. (2005) ha dirigido sus estudios a determinar la fuerza de avance axial y del par de corte presentes en el taladrado, poniendo especial énfasis en lo que ocurre en el bisel transversal de la broca.

Tradicionalmente las brocas helicoidales son reacondicionadas mediante un proceso de adelgazamiento del núcleo de la herramienta, lo que conlleva un acortamiento del bisel transversal. Según (Lin, 2007), existen algunas iniciativas para incluir en diseños originales de brocas esta disminución en la longitud del bisel transversal. Lo que propone (Lin, 2007) es un modelo matemático para determinar la disminución de la longitud del bisel transversal y realizarlo mediante una máquina rectificadora CNC. 
Lo que ha contribuido a buscar innovaciones en la geometría de las brocas, especialmente en la zona de los filos, ha sido el incrementado en el uso de los materiales compuestos en aplicaciones de la ingeniería. El proceso de taladrado ha sido uno de los puntos críticos debido a la delaminación que se produce en el material y al desgaste excesivo de la herramienta, lo que afecta la calidad y los costos de manufactura. En este sentido, (Lazar y Xirouchakis, 2013) utilizando modelos mecanicistas ensayaron dos tipos de herramientas, una broca helicoidal tradicional y una broca escariadora, pero de diámetros iguales. (Islibir y Ghassemieh, 2013) han investigado los efectos de las diferentes geometrías de las brocas, sobre la ocurrencia de la delaminación cuando se taladran materiales compuestos reforzados con fibras de carbono. (Rajmohan y Palanikumar, 2013) han experimentado sobre aleaciones de aluminio reforzadas con carburo de silicio, determinando las variables de mecanizado que inciden sobra la fuerza de avance en el taladrado, la rugosidad superficial y la formación de rebabas. (Rajmohan et al., 2012) basados en el método de Taguchi con múltiples ensayos optimizaron los parámetros de taladrado incluyendo la fuerza de avance, rugosidad superficial, desgaste de herramienta y altura de rebaba. Las conclusiones muestran que la medida del avance y el tipo de broca son los mayores y significativos factores que afectan el resultado de un proceso de taladrado.

La delaminación es reconocida como la mayor falla y defecto que se presenta después del proceso de taladrado. (Tsao, 2012), (Tsao y Chiu, 2011) han trabajado en el análisis de los factores críticos que participan en la fuerza de avance cuando que se taladran materiales compuestos. Dichos factores son la medida del avance y el paso de la hélice. En base a las técnicas de Taguchi las investigaciones de (Basavarajappa et al., 2008) conducen a establecer que la medida del avance es el principal factor que influye en la determinación de la fuerza de avance cuando se taladra sobre materiales compuestos. (Zitoune y Collombet, 2007) proponen un método numérico de predicción del valor de la fuerza de avance y que produce el defecto a la salida del agujero en un material compuesto. Estableciendo que el daño es debido al desplazamiento que sufre la broca, y que está localizado alrededor del bisel transversal. Estos autores proponen un modelo analítico para predecir la fuerza crítica de avance, tomando en cuenta la presencia de la fractura del material en la vecindad del accionar del bisel transversal. Tradicionalmente, las brocas helicoidales han tenido una geometría específica en la punta, tales como plana, cónica, cilíndrica, elipsoidal o hiperbólica, las que han sido diseñadas y adaptadas para trabajos específicos. (Sambhav et al., 2012) diseñaron una broca utilizando algoritmos de optimización, centrados en la geometría de la hélice, de los filos de corte y en el bisel transversal, cuya representación se hizo mediante los sistemas CAD, NURBS y MATLAB.

Considerando brocas con geometría convencional, (Xiong, 2009) ha propuesto una nueva metodología computacional para diseñar la curva que deberían tener los filos de las brocas helicoidales. Los parámetros considerados para obtener el nuevo perfil de la herramienta son: el ángulo de desprendimiento, el ángulo de inclinación, ángulo de posición del filo, y el ángulo de la hélice. Los resultados experimentales demostraron que las herramientas cuyos filos fueron diseñados con estas nuevas curvas, redujeron en promedio, el momento de taladrado en un $28,5 \%$ y la fuerza de avance en un $24,6 \%$ en comparación con las brocas tradicionales. Otro de los intentos por modificar la forma de la punta de las brocas con dos filos principales lo presenta (Fetecau et al., 2009), quien propuso cambiar la rectitud de los filos principales por la forma de una curva.

El afilado se realiza mediante un procedimiento de esmerilado toroidal. El bisel transversal que une a ambos filos curvos decrece su longitud en un $10 \%$ con respecto a la longitud del bisel en brocas estándar. Con esta nueva forma se disminuye el contacto del bisel con la pieza a mecanizar, lo que redunda en una disminución de las fuerza de avance y del par mecánico de taladrado. Para (Guibert et al., 2009) la mayoría de los modelos para determinar la fuerza de avance basados en la teoría de la presión específica de corte, utilizan un coeficiente de corte global. Siendo la dureza del material el único parámetro que se toma en cuenta para el cálculo de dicho coeficiente, no considerando la geometría específica de cada broca.

\section{MATERIALES Y MÉTODOS}

Todos los ensayos fueron ejecutados en una máquina-herramienta CNC, marca Hercus, modelo VMC300, de husillo vertical y máxima velocidad rotacional de 4500 (rpm). La máquina se muestra en la figura 3. Posee tres grados de libertad y las longitudes de trabajo para los distintos ejes son: $\mathrm{X}=315(\mathrm{~mm}) ; \mathrm{Y}=125$ $(\mathrm{mm})$ y $Z=185(\mathrm{~mm})$. La velocidad de avance máxima $V_{f}$, es $1296(\mathrm{~mm} / \mathrm{s})$.

Las fuerzas de avance y los torques provenientes del proceso de taladrado fueron medidos con sensores piezoeléctricos Kistler 9211B (fuerza axial) y 9329A (torque), montados en la plataforma dinamométrica, tal como lo muestra la figura 4. 


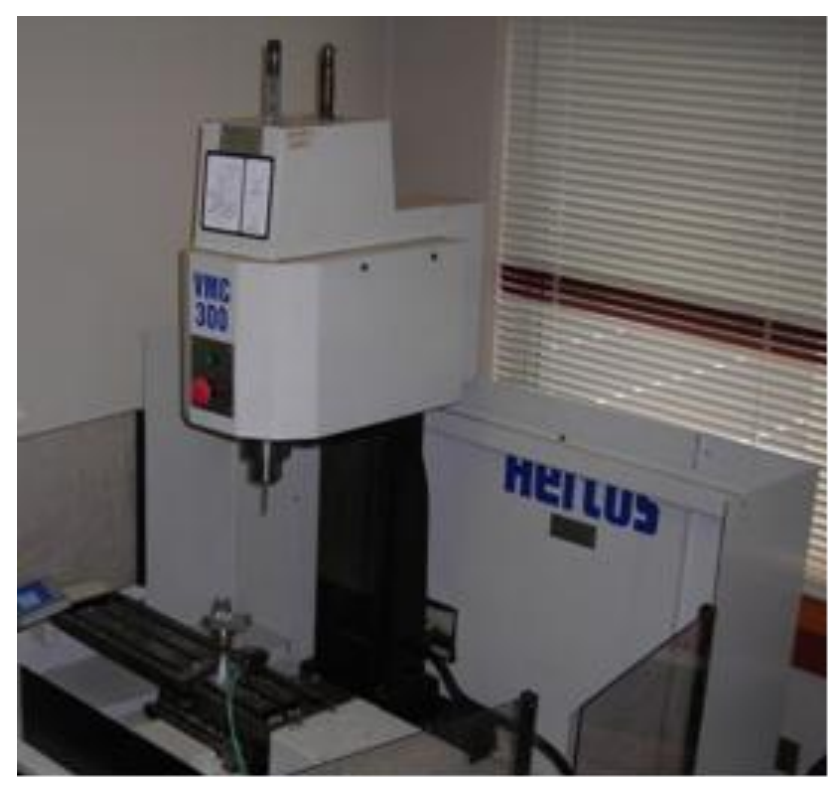

Fig.3: Máquina-herramienta CNC.

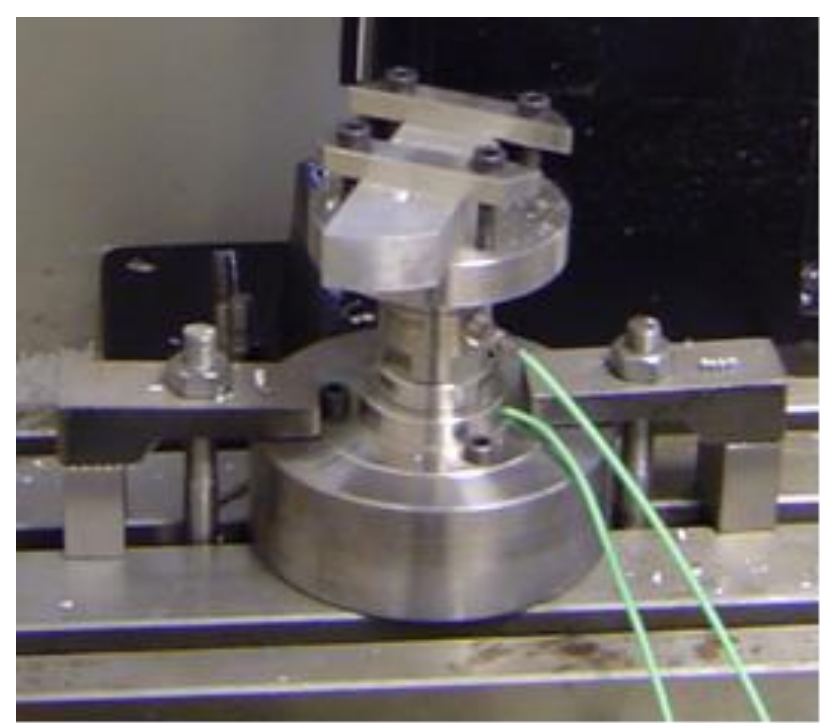

Fig.4: Plataforma dinamométrica

Para realizar las distintas actividades que contempló la presente investigación, fue necesario disponer de los siguientes recursos computacionales: ISO CNC, para programar la máquina-herramienta Hercus VMC300, ICAM Type 5073A, para operar el amplificador Kistler 5073A con salida de dos canales, NI-DAQ, para controlar la tarjeta de adquisición de datos, National Instruments 6013/14. LabView 8.1, para programar la interfaz gráfica que representa al sistema de toma de datos y Microsoft Excel, para el manejo de los datos adquiridos, su representación gráfica y el posterior almacenamiento de los mismos, en ficheros electrónicos.

El muestreo de una señal analógica lleva consigo una pérdida de datos con respecto a la señal original (Proakis y Manolakis, 2003). La frecuencia mínima de muestreo $\left(f_{s}\right)$ para poder reconstruir una señal, ha de ser al menos, el doble de la frecuencia de la señal $\left(f_{\text {máx }}\right)$ que se pretende representar, tal como lo aconseja el teorema de Nyquist-Shannon, representado por la ecuación (1). Si se utiliza una frecuencia menor a la recomendada por el mencionado teorema se produce una distorsión conocida como "solapamiento".

$f_{s} \geq 2 f_{\text {máx }}$

Para efectos de los ensayos realizados se estableció una frecuencia de muestreo constante $\left(f_{s}\right)$ de 500 (muestras/s) y un tiempo de muestreo de 4 (s), lo que origina la cantidad de 2000 (muestras) por ensayo. Otro aspecto importante a considerar es el sistema seleccionado para filtrar las señales, de modo que la elección del método de filtrado no altere en demasía la representación del proceso que se desea reproducir (Gyorki, 2004). 
Para la adquisición de los datos se hizo uso del programa en LabView, y las señales fueron filtradas digitalmente con un filtro de paso bajo con frecuencia de corte de $25 \mathrm{kHz}$. El material ensayado corresponde a una aleación de aluminio 2011, con una dureza promedio de $95 \mathrm{HB}$. Las brocas utilizadas en los ensayos son de aceros rápidos (HSS), seleccionadas a través de (Unceta, 2008).

La figura 5 muestra la configuración esquemática del proceso de mecanizado y su correspondiente circuito de adquisición de datos.

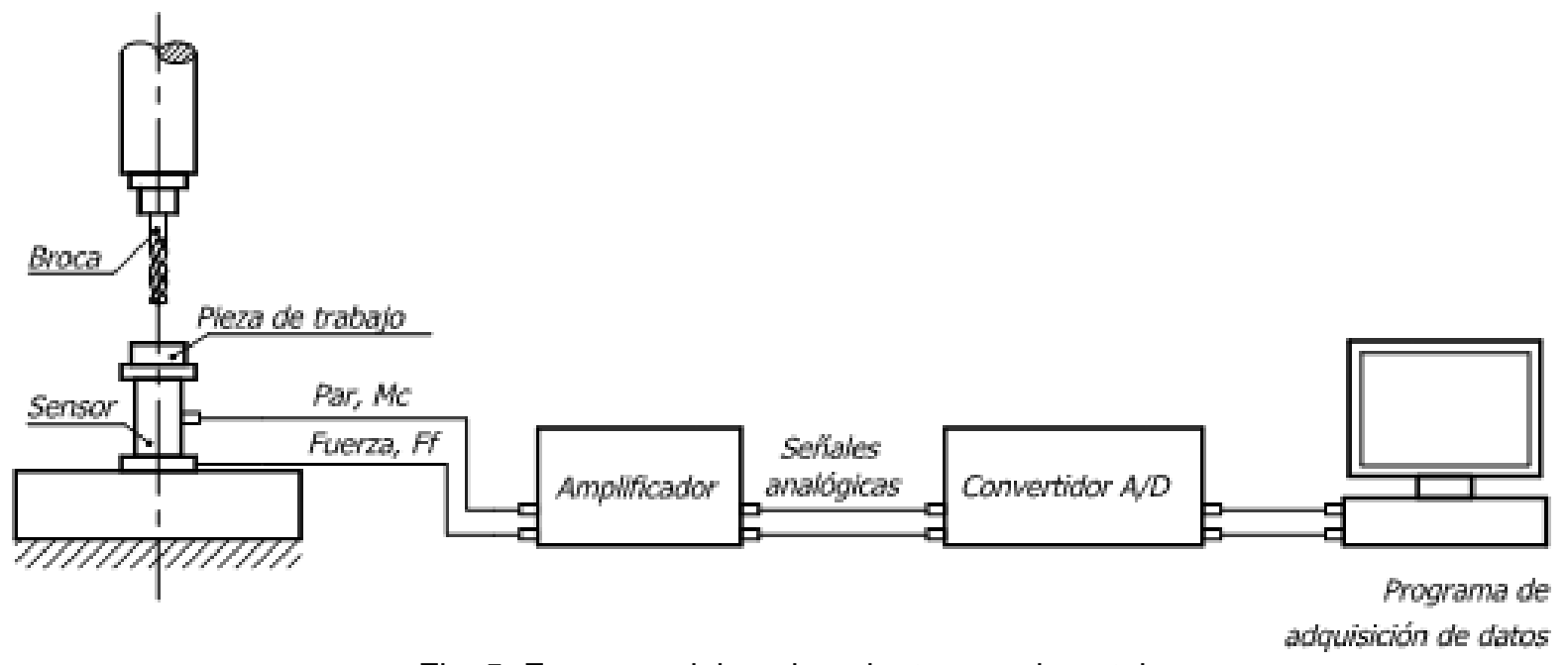

Fig. 5: Esquema del equipamiento experimental

En la Tabla 1 se encuentran las condiciones paramétricas determinadas para realizar los ensayos de taladrado, utilizando los dos tipos de brocas antes mencionados y mecanizando probetas de aluminio 2011.

Tabla 1: Parámetros de corte para los ensayos

\begin{tabular}{|c|c|c|c|c|}
\hline $\begin{array}{c}\text { Diámetro } \\
D(\mathrm{~mm})\end{array}$ & $\begin{array}{c}\text { Velocidad rotacional } \\
N(\mathrm{rev} / \mathrm{min})\end{array}$ & $\begin{array}{c}\text { Velocidad de corte } \\
V_{c}(\mathrm{~m} / \mathrm{min})\end{array}$ & $\begin{array}{c}\text { Avance axial } \\
f_{n}(\mathrm{~mm} / \mathrm{rev})\end{array}$ & $\begin{array}{c}\text { Velocidad de avance } \\
V_{f}(\mathrm{~mm} / \mathrm{min})\end{array}$ \\
\hline 4 & 3535 & 44 & 0,07 & 251 \\
5 & 2934 & 46 & 0,09 & 265 \\
6 & 2520 & 47 & 0,11 & 277 \\
7 & 2215 & 49 & 0,13 & 287 \\
\hline
\end{tabular}

La figura 6 representa a los dos tipos de brocas ensayadas, donde se destaca para cada herramienta el ángulo entre los filos principales y la altura del filo, z. De modo ilustrativo se determina que para una broca tradicional de $118^{\circ}$ entre filos principales y de diámetro $6(\mathrm{~mm})$, su altura de filo $z$ es $1,8(\mathrm{~mm})$. Respecto de la broca innovada de $140^{\circ}$ entre filos principales y de diámetro $6(\mathrm{~mm})$, su altura de filo $z$ es $1,09(\mathrm{~mm})$.
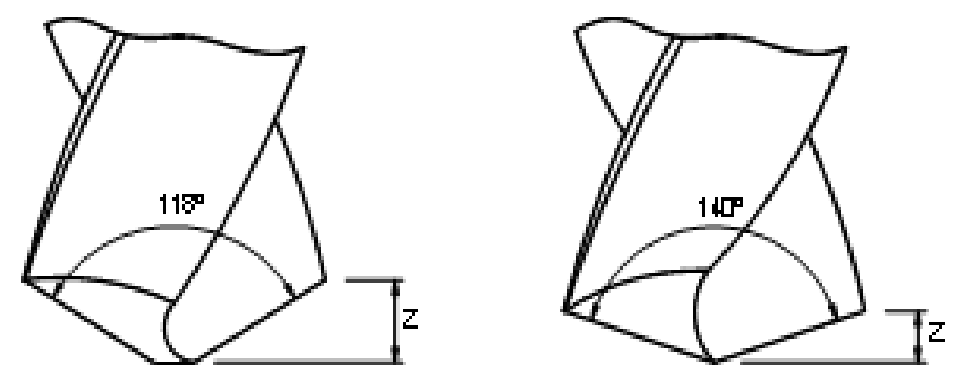

Fig. 6: Representación de los dos tipos de brocas ensayadas 


\section{RESULTADOS Y DISCUSIÓN}

La figura 7 muestra la configuración característica del comportamiento de las fuerzas de avance que se generan en la parte activa de las brocas con geometría tradicional. De modo ilustrativo, la figura 7 corresponde al taladrado de una probeta de aluminio 2011 con una broca de diámetro $6(\mathrm{~mm})$.

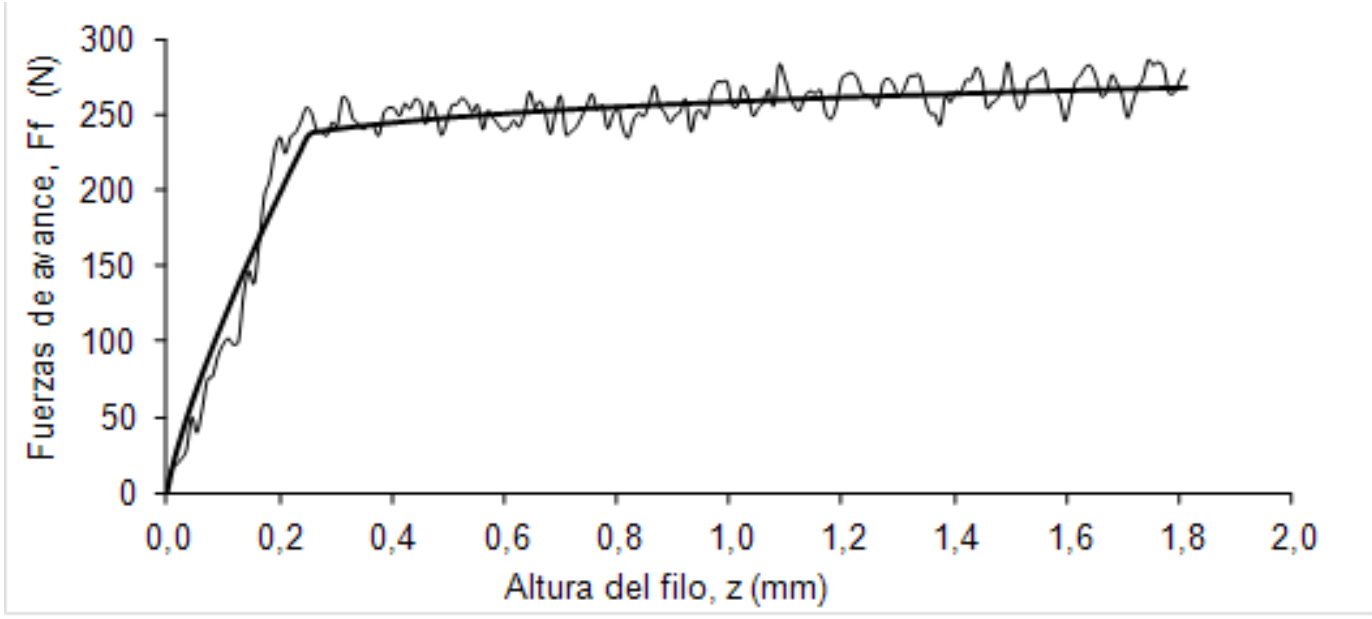

Fig.7: Fuerzas de avance en brocas tradicionales (con bisel transversal).

La figura 8 muestra la configuración característica del comportamiento de las fuerzas de avance que se generan en la parte activa de las brocas con geometría mejorada. De modo ilustrativo, la figura 8 corresponde al taladrado de una probeta de aluminio 2011 con una broca de diámetro $6(\mathrm{~mm})$.

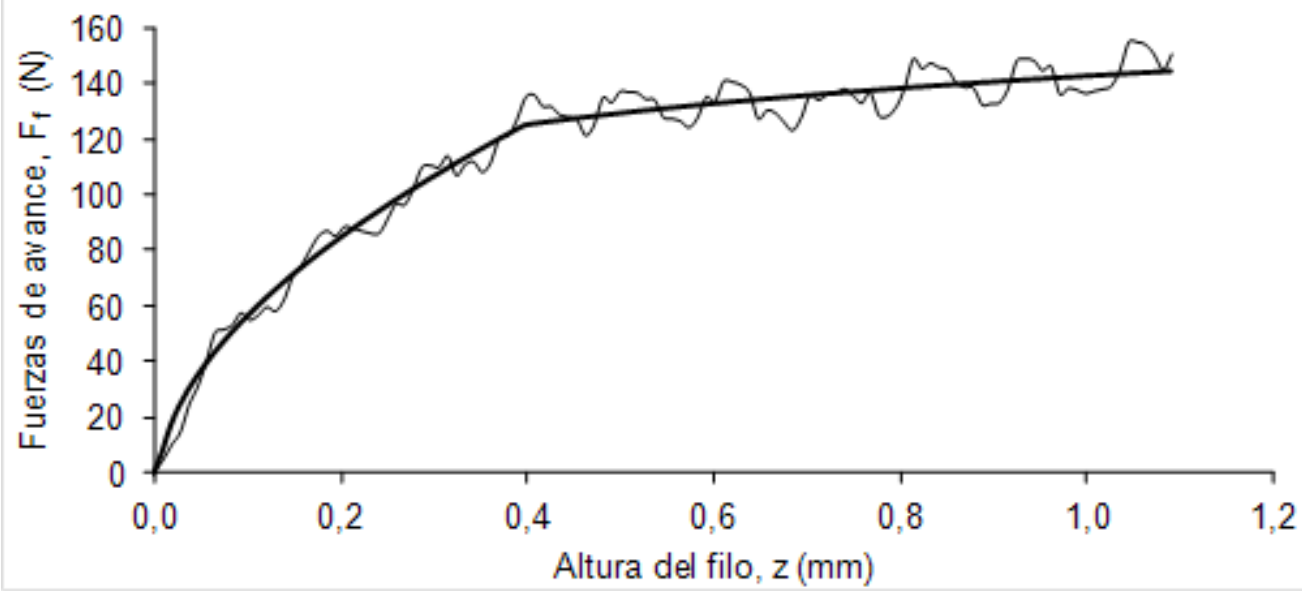

Fig.8: Fuerzas de avance en brocas mejoradas (con filo transversal).

La tabla 2 recoge los valores máximos de las fuerzas de avance axial en un proceso de taladrado cuando se utilizan brocas con geometría tradicional (bisel transversal) y brocas con geometría mejorada (filo transversal). Asociado a estas herramientas, se muestra el decremento y porcentaje de variación que se produce entre las fuerzas máximas de los dos tipos de brocas estudiadas.

Tabla 2: Fuerzas máximas de avance en taladrado

\begin{tabular}{|c|c|c|c|c|}
\hline $\begin{array}{c}\text { Diámetro } \\
\mathrm{D}(\mathrm{mm})\end{array}$ & $\begin{array}{c}\text { Brocas con bisel } \\
\text { transversal }\end{array}$ & $\begin{array}{c}\text { Brocas con filo } \\
\text { transversal }\end{array}$ & $\begin{array}{c}\text { Decremento } \\
\text { de fuerza }\end{array}$ & $\begin{array}{c}\text { Porcentaje de } \\
\text { decremento }\end{array}$ \\
\cline { 2 - 5 } & $\mathrm{F}_{\mathrm{f}(\mathrm{b})}(\mathrm{N})$ & $\mathrm{F}_{\mathrm{f}(\mathrm{f})}(\mathrm{N})$ & $\Delta \mathrm{F}(\mathrm{N})$ & $\%$ \\
\hline 4 & 121,85 & 86,71 & 35,14 & 28,8 \\
5 & 199,24 & 122,56 & 76,68 & 38,5 \\
6 & 276,63 & 158,41 & 118,22 & 42,7 \\
7 & 354,02 & 194,26 & 159,76 & 45,1 \\
\hline
\end{tabular}


La figura 9 representa la tendencia lineal que experimentan los dos tipos de herramientas utilizadas, cuando se grafica la fuerza de avance axial máxima que genera cada grupo de brocas ensayadas, y que son representativas de cada diseño geométrico de las herramientas. Los resultados para ambos tipos de herramientas evidencian una tendencia lineal creciente entre sí y divergente entre ambas herramientas.

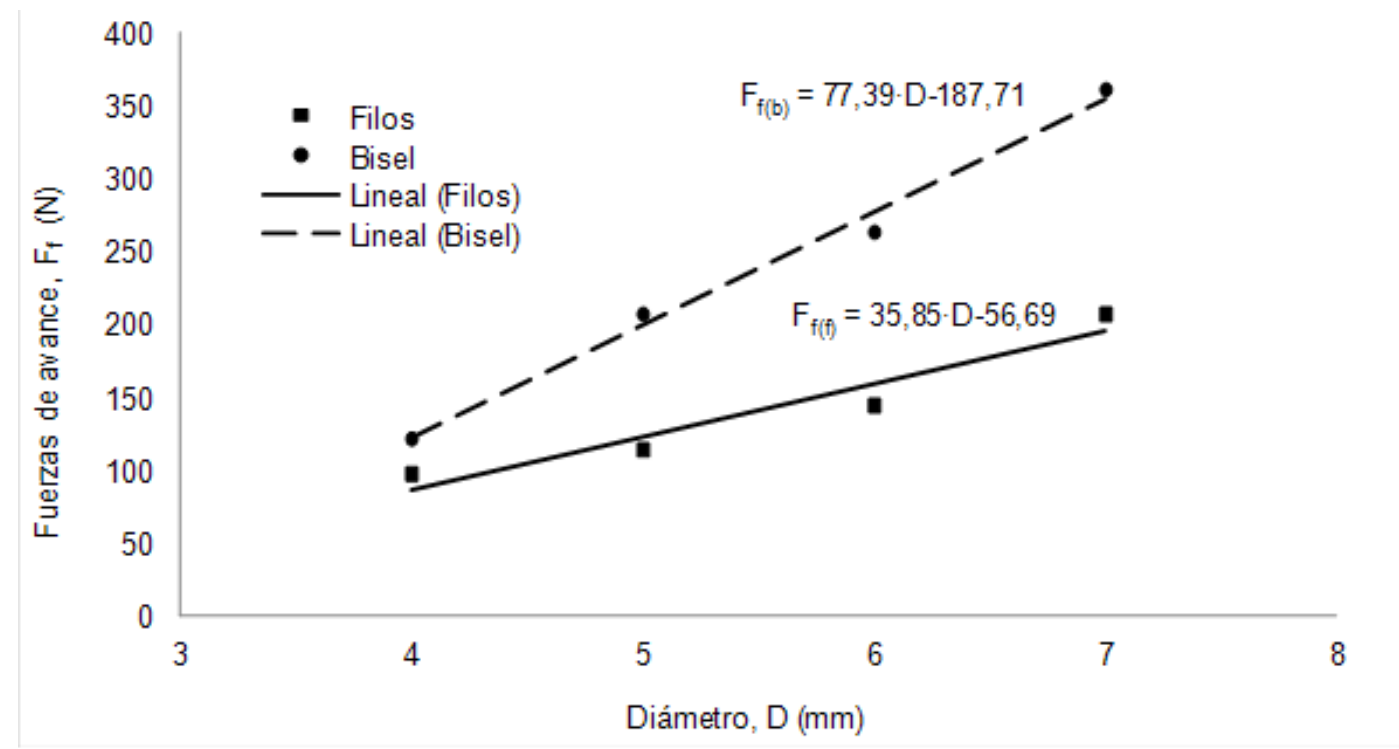

Fig.9: Fuerzas máximas de avance para brocas con bisel y con filo transversal.

\section{CONCLUSIONES}

De acuerdo a los resultados y la discusión expuesta, se presentan las principales conclusiones:

i) Bajo las mismas condiciones de taladrado y mecanizando un mismo material, las brocas con geometría tradicional que incluyen el bisel transversal requieren una mayor fuerza de avance axial, que las brocas con geometría mejorada en la parte activa, destacando la presencia del bisel transversal que se divide en dos filos de corte.

ii) En la medida que va creciendo el diámetro de las brocas y aplicando las condiciones de corte recomendadas para un proceso de taladrado que le corresponde a cada diámetro, se verifica que el comportamiento individual, tanto de las brocas tradicionales como las de geometría mejorada, tienen individualmente una tendencia lineal creciente, pero que resultan divergentes entre ellas.

iii) La geometría mejorada en la punta de la broca, con presencia del filo transversal, permite una acción de autocentrado, con lo que se mejora ostensiblemente el rendimiento y calidad del taladrado. Perforar con este tipo de brocas evita realizar el proceso de pretaladrado para lograr buenos resultados.

iv) El bisel de las brocas tradicionales no actúa como un filo en el proceso de corte, sino en un proceso de indentación, similar a la deformación de material que se produce al realizar un ensayo de dureza.

\section{AGRADECIMIENTOS}

Los autores desean agradecer a la Dirección de Investigación de la Universidad de La Frontera, que mediante el proyecto DIUFRO DI10-0012 financió este trabajo de investigación.

\section{REFERENCIAS}

Anish, P., S.G Kapoor y R.E. De Vor, A Chisel Edge Model for Arbitrary Drill Point Geometry, Journal of Manufacturing Science and Engineering: (127) 23-32 (2005).

Audy, J., A Study of Computer Assisted Analysis of Effects of Drill Geometry and Surface Coating on Force and Power in Drilling, Journal of Materials Processing Technology (204) 130-138 (2008).

Basavarajappa, S., G. Chandramohan. y J.P. Davim, Some Studies on Drilling of Hybrid Metal Matrix Composites Based on Taguchi Techniques, Journal of Material Processing Technology, (196) 332-338 (2008). 
Fetecau, C., Stan, F. y Oancea, N, Toroidal grinding method for curved cutting edge twist drills. Journal of Materials Processing Technology. (209) 3460-3468 (2009).

Gong, Y., C. Lin y K.F. Ehmann, Dynamic of Initial Penetration in Drilling: Part 1- Mechanistic Model for Dynamic Forces", Journal of Manufacturing Science and Engineering: (127), 280-288 (2005).

Guibert, N., H. Paris. y J. Rech, Identification of Thrust Force Models for Vibratory Drilling, International, Journal of Machine Tools \& Manufacture (49) 730-738 (2009).

Gyorki, J. R., Signal Conditioning \& PC-Based Data Acquisition, lotech, Inc. USA. (2004).

Hamade, R.F., C.Y. Seif y F. Ismail, Extracting Cutting Force Coefficients from Drilling Experiments, International Journal of Machine Tools \& Manufacture: (46) 387-396 (2006).

Isbilir, O. y E. Ghassemieh, Numerical Investigation of Effect of Drill Geometry on Drilling Induced Delamination of Carbon Fiber Reinforced Composites, Composites Structures: (105) 126-133 (2013).

Lazar, M-B. y P. Xirouchakis, Mechanical Load Distribution along the main cutting edges in drilling, Journal of Materials Processing Technology: (213) 245-260 (2013).

Lin, P.D. y C.S. Tzeng, New method for Determination of the Pose of the Grinding Wheel for Thinning Drill Point, International Journal of Machine Tools \& Manufacture (47) 2218-2229 (2007).

Oxford, C. Jr. y M. Rochester, On the Drilling of Metals 1. Basic Mechanics of the Process, Transactions of the ASME: (77), 103-114 (1955).

Pirtini, M., I. Lazoglu, Forces and Quality in Drilling, International Journal of Machine Tools \& Manufacture: (45), 1271-1281 (2005).

Proakis, J.G. y D.G. Manolakis, Tratamiento Digital de Señales, Principios, Algoritmos y Aplicaciones", Prentice Hall, Madrid, (2003).

Rajmohan, T. y K. Palanikumar, Application of the Central Composite Design in Optimization of Machining Parameters in Drilling Hybrid Metal Matrix Composites, Measurement (46) 1470-1481 (2013).

Rajmohan, T., K. Palanikumar, y M. Kathirvel, Optimization of Machining Parameters in Drilling Hybrid Aluminium Metal Matrix Composites, Transactions of Nonferrous Metals Society of China (22) 1286-1297 (2012).

Sambhav, K., P. Tandon. y S.G. Dhande, Geometric Modeling and Validation of Twist with a Generic Point Profile, Applied Mathematical Modeling (36) 2384-2403 (2012).

Shaw, M.C., C.Jr. Oxford, On the Drilling of Metals 2- The Torque and Thrust in Drilling, Transactions of the ASME: (79) 139-148 (1957).

Tsao, C.C, Effect of Induced Bending Moments (IBM) on Critical Thrust Force for Delamination in Step Drilling of Composites, International Journal of Machine Tools \& Manufacture (59) 1-5 (2012).

Tsao, C.C. y Y.C. Chiu, Evaluation of Drilling Parameters on Thrust Force in Drilling Carbon Fiber Reinforced Plastic (CFRP) Composite Laminates Using Compound Core-Special Drills, International Journal of Machine Tools \& Manufacture (51) 740-744 (2011).

UNCETA, Catálogo de herramientas de calidad, http://www.unceta.com/catalogos_unceta.asp (2008)

Wang, J. y Q. Zhang, A Study of High-Performance Plane Rake Faced Twist Drills. Part I: Geometrical Analysis and Experimental Investigation, Inter. J. of Machine Tools \& Manufacture: (48) 1276-1285 (2008).

Xiong, L., N. Fang. y H. Shi, A New Methodology for Designing a Curve-Edge Twist Drill whit an Arbitrary Given Distribution of the Cutting Angles Along the Tool Cutting Edge, International Journal of Machine Tools \& Manufacture (49) 667-677 (2009).

Zitoune, R. y F. Collombet, Numerical Prediction of the Thrust Force Responsible of Delamination During the Drilling of the Long-Fibre Composite Structures. Composites: Part A (38) 858-866 (2007). 